\title{
AN AR-MAP WHOSE RANGE IS MORE INFINITE-DIMENSIONAL THAN ITS DOMAIN
}

\author{
J. J. DIJKSTRA, J. VAN MILL, AND J. MOGILSKI
}

(Communicated by James E. West)

\begin{abstract}
We construct an example of an AR-map $f: X \rightarrow Y$, where $X$ is a strongly countable dimensional compact AR and $Y$ is a countable dimensional AR which is not strongly countable dimensional. Using this map we find a shrinkable decomposition of the pre-Hilbert space $l_{f}^{2}$ whose quotient map does not stabilize to a near homeomorphism. We also present a partial result concerning the question whether cell-like maps preserve countable dimensionality.
\end{abstract}

\section{INTRODUCTION}

A space $X$ is (strongly) countable aimensional if it is a countable union of (closed) finite-dimensional subspaces. The aim of this note is to construct an example of an AR-map $f: X \rightarrow Y$, where $X$ is a strongly countable dimensional compact AR and $Y$ is a countable dimensional compact AR which is not strongly countable dimensional. Observe that the range of our map is "more infinite-dimensional than its domain." The simplicity of our example shows that this phenomenon has nothing to do with dimension raising cell-like maps (Dranišnikov [4]). This example can be used to find a shrinkable AR-map whose domain is the pre-Hilbert space

$$
l_{f}^{2}=\left\{\left(x_{i}\right)_{i} \in l^{2}: x_{i}=0 \text { for all but finitely many } i\right\},
$$

but that does not stabilize to a near homeomorphism. This answers several questions of J. P. Henderson (see West [13]). In the final section we discuss the problem whether cell-like maps between compact ANRs preserve countable dimensionality.

All spaces are assumed to be separable metric. As usual, I denotes the interval $[0,1]$.

\section{THE BASIC EXAMPLE}

Proposition 2.1. There exists an AR-map f from a strongly countable dimensional compact AR $X$ onto a countable dimensional but not strongly countable dimensional compact $A R Y$.

Received by the editors June 19, 1990.

1980 Mathematics Subject Classification (1985 Revision). Primary 54G20, 57N20. 
Proof. The construction is inspired by the classic examples of nonstrongly countable dimensional spaces, see [6, Example 1.12]. First, we construct the range of the map $f$. Let $D$ denote a disk. Pick a null-sequence $\left\{J_{k}\right\}_{k=1}^{\infty}$ consisting of pairwise disjoint arcs in $D$ the union of which is dense. For every $k$, let $f_{k}: J_{k} \rightarrow I^{k}$ be a continuous surjection with finite fibres. Standard examples of space-filling curves have this property. We recall the construction of one such map. Define the homeomorphism $h$ between the Cantor sets $3^{\mathbf{N}}=\prod_{i=1}^{\infty}\{0,1,2\}$ and $3^{\mathbf{N}} \times 3^{\mathbf{N}}$ by $h(\varepsilon)=(\alpha, \beta)$, where

and

$$
\alpha_{i}= \begin{cases}\varepsilon_{2 i-1}, & \text { for } \sum_{j<i} \beta_{i} \text { even, } \\ 2-\varepsilon_{2 i-1}, & \text { for } \sum_{j<i} \beta_{i} \text { odd }\end{cases}
$$

$$
\beta_{i}= \begin{cases}\varepsilon_{2 i}, & \text { for } \sum_{j \leq i} \alpha_{i} \text { even, } \\ 2-\varepsilon_{2 i}, & \text { for } \sum_{j \leq i} \alpha_{i} \text { odd. }\end{cases}
$$

Let $q: 3^{\mathbf{N}} \rightarrow I$ stand for the usual quotient map $q(\varepsilon)=\sum_{i=1}^{\infty} \varepsilon_{i} 3^{-i}$. Since the fibres of $q$ contain at most two points, the fibres of $(q \times q) \circ h$ contain at most four points. One readily verifies that $q(\varepsilon)=q\left(\varepsilon^{\prime}\right)$ implies $(q \times q) \circ h(\varepsilon)=$ $(q \times q) \circ h\left(\varepsilon^{\prime}\right)$. So there exists a continous map $p: I \rightarrow I \times I$ with $(q \times q) \circ h=$ $p \circ q$. Then $p$ is obviously surjective and its fibres consist of at most four points. The functions $f_{k}$ are obtained by iterating $p$.

Put

$$
\mathscr{G}=\left\{\left\{y: y=x \text { or } f_{k}(y)=f_{k}(x) \text { for some } k \in \mathbf{N}\right\}: x \in D\right\} .
$$

Then $\mathscr{G}$ is an upper semicontinuous decomposition of $D$, so we can consider the quotient space $Y=D / \mathscr{G}$ with corresponding quotient map $\pi: D \rightarrow Y$. Observe that $Y$ is obtained from $D$ by replacing each $J_{k}$ by $I^{k}$. It clearly follows that $Y$ is countable dimensional. We claim that $Y$ is not strongly countable dimensional. This will follow by a standard argument. Let $U \subset Y$ be nonempty and open. Then $\pi^{-1}(U)$ is open in $D$ and nonempty so it contains infinitely many $J_{k}$ 's. This implies that $U$ contains cells of arbitrarily large dimension, i.e., $\operatorname{dim} U=\infty$. Now assume that $Y=\bigcup_{i=1}^{\infty} A_{i}$, with each $A_{i}$ closed. Then by the Baire Category Theorem there exists an $i$ such that $A_{i}$ has nonempty interior and hence we have $\operatorname{dim} A_{i}=\infty$. We conclude that $Y$ is not strongly countable dimensional. By a direct argument, it is easily seen that $Y$ is an AR. Alternatively, apply Kozlowski [10, Theorem 15]: the image of an ANR under a map $f$ that is determined on a null-sequence of ARs $A_{i}$ such that each $f\left(A_{i}\right)$ is an AR, is again an ANR.

We now look at the domain. Define

$$
Z=(D \times\{0\}) \cup \bigcup_{k=1}^{\infty} J_{k} \times[0,1 / k] .
$$

It is easy to see that $Z$ is a closed subspace of $D \times I$. Observe that the collection $\left\{J_{k} \times\left\{\frac{1}{k}\right\}\right\}_{k=1}^{\infty}$ is a null sequence in $Z$. For every $k$ let $g_{k}: J_{k} \times\left\{\frac{1}{k}\right\} \rightarrow I^{k}$ be defined by $g_{k}\left(x, \frac{1}{k}\right)=f_{k}(x)$, i.e., $g_{k}$ is a copy of the map $f_{k}$. Now consider the collection

$$
\mathscr{H}=\left\{\left\{y: y=x \text { or } g_{k}(y)=g_{k}(x) \text { for some } k \in \mathbf{N}\right\}: x \in Z\right\} .
$$

Then $\mathscr{H}$ is an upper semicontinuous decomposition of $Z$, so we can consider the quotient space $X=Z / \mathscr{H}$ with corresponding quotient map $\rho: Z \rightarrow X$. 
Observe that $X$ is a disc with the mapping cylinders of the maps $f_{k}$ attached to it. Indeed, for every $k$ we have that the set

$$
\rho\left(J_{k} \times[0,1 / k]\right)
$$

is a canonical copy of the mapping cylinder of $f_{k}$. Observe that $X$ is an AR for the same reason as $Y$ is. Also, observe that $Z$ is strongly countable dimensional. If we remove $\bigcup_{k=1}^{\infty} \rho\left(J_{k} \times\left\{\frac{1}{k}\right\}\right)$ from $Z$ then what remains is (homeomorphic to) a $\sigma$-compact subspace of $D \times I$.

Finally, we define the map $f$. For every $k$ let $c_{k}: \rho\left(J_{k} \times\left[0, \frac{1}{k}\right]\right) \rightarrow I^{k}$ be the collapse to the base. Then $c_{k}$ is clearly an AR-map (its point inverses are cones of finite sets). Put

$$
\mathscr{I}=\left\{\left\{y: y=x \text { or } c_{k}(y)=c_{k}(x) \text { for some } k \in \mathbf{N}\right\}: x \in X\right\} .
$$

Then $\mathscr{I}$ is upper semicontinous, and the decomposition space $X / \mathscr{I}$ is clearly homeomorphic to $Y$. Since the quotient map $X \rightarrow X / \mathscr{I}$ is an AR-map, we are done.

For a $\sigma$-compact space $X$, let $\gamma(X)$ be the minimum ordinal $\alpha$ such that $X$ is a countable union of compact subsets with transfinite dimension ind $<\alpha$ (cf. [2, p. 282]). Let us recall that the transfinite dimension ind is the extension by transfinite induction of the classical Menger-Urysohn inductive dimension (ind $X=-1$ means $X=\varnothing$, ind $X \leq \alpha$, where $\alpha$ is a countable ordinal, if and only if $X$ has a open basis consisting of sets $B$ with ind $(\partial B)<\alpha)$. Obviously, we have $\gamma(X) \leq$ ind $X$ and a compactum $X$ is strongly countable dimensional if and only if $\bar{\gamma}(X) \leq \omega_{0}$. In Proposition 2.1 we constructed an AR-map of a compact AR $X$, with $\gamma(X)=\omega_{0}$, onto a compact AR $Y$, with $\gamma(Y)=\omega_{0}+1$. By a slight modification of our construction we obtain:

Proposition 2.2. For every countable ordinal $\alpha, \alpha \geq \omega_{0}$, there exists an AR-map $f_{\alpha}$ from a strongly countable dimensional compact $A R X_{\alpha}$ onto a compact $A R$ $Y_{\alpha}$ with $\gamma\left(Y_{\alpha}\right) \geq \alpha$.

Proof. As in the proof of Proposition 2.1, we pick a null-sequence $\left\{J_{k}\right\}_{k=1}^{\infty}$ consisting of pairwise disjoint arcs in a disk $D$. Let $H^{\alpha}$, for $\alpha<\omega_{1}$, stand for a slight modification of Henderson's "transfinite cubes." We define $H^{\alpha}$ together with a point $p^{\alpha} \in H^{\alpha}$ as follows. $H^{0}=\{0\}, H^{\alpha+1}=H^{\alpha} \times I$, and $p^{\alpha+1}=\left(p^{\alpha}, 0\right)$. If $\alpha$ is a limit ordinal then we write $\{\beta: \beta<\alpha\}=\left\{\beta_{i}: i \in \mathbf{N}\right\}$ and we connect in the topological sum $\bigoplus_{i=1}^{\infty} H^{\beta_{i}}$ every $p^{\beta_{i}}$ with $p^{\beta_{i+1}}$ by an arc. We then take the one point compactification of the resulting space. This is $H^{\alpha}$ and $p^{\alpha}$ is the point at infinity. The space $H^{\alpha}$ is a strongly countable dimensional compact $\mathrm{AR}$ with ind $=\alpha$ (see [8]).

Now, let $\alpha_{k}$ be a nondecreasing sequence converging to $\alpha$. For every $k$, let $g_{k}: J_{k} \rightarrow H^{\alpha_{k}}$ be a continuous surjection with finite fibres (it is not hard to construct $g_{k}$ using the map $p$ described in the proof of Proposition 2.1). We construct the spaces $X_{\alpha}, Y_{\alpha}$ and the map $f_{\alpha}$ in the same way as $X, Y$, and $f$ were constructed. Hence $X_{\alpha}$ and $Y_{\alpha}$ are compact ARs and $f_{\alpha}$ is an AR-map. By strong countable dimensionality of $H^{\alpha_{k}}$, for $k=1,2, \ldots, X_{\alpha}$ is strongly countable dimensional. Since each nonempty subset of $Y_{\alpha}$ contains infinitely many $H^{\alpha_{k}}$ 's we have $\gamma\left(Y_{\alpha}\right) \geq \alpha$. 


\section{A SHRINKABLE DECOMPOSITION OF $l_{f}^{2}$}

The pre-Hilbert space $l_{f}^{2}$ is strongly countable dimensional and universal for all strongly countable dimensional compacta. Henderson and Walsh [9] constructed a cell-like map $f$ from $l_{f}^{2}$ onto an AR $M$ such that $M$ is not homeomorphic to $l_{f}^{2}$ but $M \times \mathbf{R} \cong l_{f}^{2}$. Proposition 2.1 allows us to construct an AR-map of $l_{f}^{2}$ onto an AR $Z$ such that not even $Z \times l_{f}^{2}$ is homeomorphic to $l_{f}^{2}$. Our map has another interesting property. Let us recall that a proper map $f: X \rightarrow Y$ is shrinkable if for every open cover $\mathscr{U}$ of $Y$ and every open cover $\mathscr{V}$ of $X$ there exists a homeomorphism $h: X \rightarrow X$ such that $f \circ h$ is $\mathscr{U}$-close to $f$ and $\left\{h\left(f^{-1}(y)\right)\right\}_{y \in Y}$ refines $\mathscr{V}$. Bing's Shrinking Criterion states that if $X$ is complete and $f$ is shrinkable, then $Y$ is homeomorphic to $X$ and $f$ is a near homeomorphism. The following proposition shows that the completeness assumption is essen:ial and it answers questions NCL 12, 13, and 14 in West [13].

Proposition 3.1. There exists a shrinkable AR-map from $l_{f}^{2}$ onto an absolute retract $Z$ such that $Z \times l_{f}^{2}$ is not homeomorphic to $l_{f}^{2}$.

Proof. Let $X$ be the strongly countable dimensional AR from Proposition 2.1. We may assume that $X$ is a subset of $l_{f}^{2}$. By compactness $X$ is a Z-set in $l_{f}^{2}$. If $f: X \rightarrow Y$ is the AR-map from Proposition 2.1, then the decomposition

$$
\mathscr{F}=\left\{f^{-1}(y): y \in Y\right\} \cup\left\{\{x\}: x \in l_{f}^{2} \backslash X\right\}
$$

is upper semicontinuous and the quotient map $F: l_{f}^{2} \rightarrow l_{f}^{2} / \mathscr{F}$ is an AR-map. By [10] the quotient space $Z=l_{f}^{2} / \mathscr{F}$ is an AR. Of course, $Z$ and $Z \times l_{f}^{2}$ contain topological copies of the space $Y$ which is not strongly countable dimensional. Hence, $Z \times l_{f}^{2}$ is not homeomorphic to the strongly countable dimensional space $l_{f}^{2}$. We prove that $F$ is shrinkable. Let $\mathscr{U}$ and $\mathscr{V}$ be open covers of $Z$ and $l_{f}^{2}$, respectively. Since $F$ is a fine homotopy equivalence (see [7] or Theorem 4.2) there exists a map $G: Z \rightarrow l_{f}^{2}$ such that $G \circ F$ is $F^{-1}(\mathscr{U})$-homotopic to the identity. The map $G \circ F \mid X$ can be approximated by an embedding $v: X \rightarrow l_{f}^{2}$ such that $v$ is $F^{-1}(\mathscr{U})$-homotopic and $\mathscr{V}$-close to $G \circ F \mid X$. Since $X$ and $v(X)$ are $Z$-sets there exists a homeomorphism $h: l_{f}^{2} \rightarrow l_{f}^{2}$ which is $F^{-1}$ (st $\mathscr{U}$ )-homotopic to the identity and such that $h \mid X=v$. Thus $F \circ h$ and $F$ are st $\mathscr{U}$-close. Since $h \mid X$ is $\mathscr{V}$-close to $G \circ F$ and $G \circ F\left(F^{-1}(y)\right)$ is a singleton for all $y \in Y$ the family $\left\{h\left(F^{-1}(y)\right): y \in Y\right\}$ refines st $\mathscr{V}$.

\section{TRANSFINITE DIMENSION AND CELL-LIKE MAPS}

The spaces in this section are assumed to be compact. The following question is closely related to the example of $\S 2$.

Question 4.1. Let $f: X \rightarrow Y$ be a cell-like map of a countable dimensional ANR $X$ onto an ANR $Y$. Is $Y$ countable dimensional?

Let us recall that a cell-like map $f: X \rightarrow Y$ between absolute neighborhood retracts is a fine homotopy equivalence (i.e., for every open cover $\mathscr{U}$ of $Y$ there exists a map $g: Y \rightarrow X$ such that $f \circ g$ is $\mathscr{U}$-homotopic to id $Y$ and $g \circ f$ 
is $f^{-1}(U)$-homotopic to id $\left.X\right)$ and a hereditary shape equivalence (i.e., for each ANR $Z$ the map $f$ produces a one-to-one correspondence between the homotopy classes of $C(Y, Z)$ and $C(X, Z))$. Hereditary shape equivalence is the natural extension to arbitrary compacta of fine homotopy equivaience between ANRs. Let us have a closer look at this. Let $X$ be a countable dimensional compactum and let $f: X \rightarrow Y$ be a cell-like map. By the Freudenthal Expansion Theorem (see [3]) $X$ is the inverse limit of finite dimensional ANRs, say $X=\lim \left\{X_{n}, f_{n}\right\}$, with each $X_{n}$ ANR. Let $\tilde{X}$ be the infinite mapping cylinder of the sequence $\left\{X_{n}, f_{n}\right\}_{n=1}^{\infty}$ with a copy of $X$ attached at its end. Then $\tilde{X} \in$ ANR and $\tilde{X}$ is countable dimensional (observe that we added a countable dimensional set to $X)$. Let $\mathscr{G}_{f}=\left\{f^{-1}(y): y \in Y\right\} \cup\{$ points $\}$, then $\mathscr{G}_{f}$ is a cell-like decomposition of $\widetilde{X}$ and the quotient map $\tilde{f}: \widetilde{X} \rightarrow \widetilde{X} / \mathscr{G}_{f}$ is a cell-like map. Of course $Y$ embeds in $\tilde{X} / \mathscr{G}_{f}$. Let us recall the following theorem of Kozlowski [10] (see also [5, p. 133]).

Theorem 4.2. The following statements are equivalent:

(1) $f$ is a hereditary shape equivalence;

(2) $\widetilde{X} / \mathscr{G}_{f} \in A N R$

(3) $\tilde{f}$ is a fine homotopy equivalence;

(4) $\tilde{f}$ is a hereditary shape equivalence.

By Theorem 4.2, Question 4.1 is equivalent to the following question (posed by Henderson, Kozlowski, and Walsh at the problem session of the AMS meeting in Norman, 1983).

Question 4.3. Do hereditary shape equivalences preserve countable dimensionality?

A complete space $X$ is countable dimensional if and only if ind $X$ exists. Because hereditary shape equivalences do not raise finite dimension we ask

Question 4.4. Let $f: X \rightarrow Y$ be a hereditary shape equivalence between countable dimensional compacta. Is ind $Y \leq$ ind $X$ ?

Ancel [1] proved that a cell-like map with a countable dimensional range is a hereditary shape equivalence. We define

$\eta(X)=\sup \{$ ind $Y: Y$ is a countable dimensional cell-like image of $X\}$.

We present the following partial result concerning Question 4.3.

Proposition 4.5. If for every countable dimensional compactum $X$ we have $\eta(X)$ $<\omega_{1}$, then hereditary shape equivalences preserve countable dimensionality.

Proof. Let $f: X \rightarrow Y$ be a hereditary shape equivalence of a countable dimensional compactum $X$ onto $Y$. In order to prove that $Y$ is countable dimensional we shall apply Pol's characterization of countable dimensionality (see [12]) but we modify his orginal construction slightly. Let $Y=\lim \left\{Y_{n}, g_{n}\right\}$, where each $Y_{n}$ is a finite dimensional ANR. As in the beginning of this section, $\widetilde{Y}$ stands for the infinite mapping cylinder of the sequence $\left\{Y_{i}, g_{i}\right\}_{i=1}^{\infty}$ with the copy of $Y$ attached at its end. If $\widetilde{Y}_{n}$ is a finite mapping cylinder of the finite sequence $\left\{Y_{i}, g_{i}\right\}_{i=1}^{n}$ and if $\pi_{n}$ is the natural collapse of $\widetilde{Y}$ onto $\widetilde{Y}_{n}$, then 
$\tilde{Y}_{n}$ is a finite dimensional ANR, $\pi_{n}$ is a cell-like map which is a hereditary shape equivalence and the sequence $\delta_{n}=\sup _{z \in \widetilde{Y}_{n}} \operatorname{diam}\left(\pi_{n}^{-1}(z)\right)$, converges to 0 . Let $C$ be the Cantor set and let $C_{0}=\left\{c_{1}, c_{2}, \ldots\right\}$ be a dense countable subset of $C$. We shall denote by $\mathscr{P}$ the upper semicontinuous decomposition of the space $C \times \widetilde{Y}$ into singletons $\{(c, y)\}$, where $c \notin C_{0}$, and the sets $\left\{c_{i}\right\} \times \pi_{i}^{-1}(z)$, where $z \in \widetilde{Y}_{i}$ and $i=1,2, \ldots$. The space $C \times \widetilde{Y} / \mathscr{P}$ is compact and the quotient map $\pi: C \times \widetilde{Y} \rightarrow C \times \widetilde{Y} / \mathscr{P}$ is a hereditary shape equivalence. According to Pol's characterization the space $\widetilde{Y}$ is countable dimensional if and only if

$$
\sup _{D \in \widehat{C}_{0}} \operatorname{ind}(\pi(D \times \tilde{Y}))<\omega_{1},
$$

where $\widehat{C_{0}}=\left\{D: D\right.$ is a compact subset of $\left.C_{0}\right\}$. Now, let $\widehat{X}$ be a compactum which is the infinite mapping cylinder of the sequence $\left\{Y_{n}, g_{n}\right\}$ with a copy of the space $X$ attached at its end (the compactum $\hat{X}$ can be constructed as follows: assume that $X \subset I^{\omega} \times\{0\} \subset I^{\omega} \times[0,1]$, then the quotient space $I^{\omega} \times[0,1] / \mathscr{F}$ of the decomposition $\mathscr{F}=\{\{(q, t)\}: t \in(0,1]\} \cup$ $\{\{(q, 0)\}:(q, 0) \notin X\} \cup\left\{f^{-1}(y): y \in Y\right\}$, is homeomorphic to $I^{\omega} \times[0,1]$ (see [11, Lemma 7.5.2]) and $\hat{f}(X)$ is a $Z$-set, where $\hat{f}$ is the quotient map; we set $\widehat{X}=\hat{f}^{-1}(\widetilde{Y})$ assuming that $\widetilde{Y}$ is a subset of $I^{\omega} \times[0,1] / \mathscr{F}$ whose intersection with $\hat{f}\left(I^{\omega} \times\{0\}\right)$ is $\left.Y\right)$. By the construction $\hat{X}$ is countable dimensional, $\hat{f} \mid X=f$ and $\hat{f}$ maps $\widehat{X} \backslash X$ onto $\widetilde{Y} \backslash Y$. Then $\phi=\operatorname{id}_{C} \times(\hat{f} \mid \hat{X})$ is a hereditary shape equivalence of $C \times \widehat{X}$ onto $C \times \tilde{Y}$. Thus $\pi \circ \phi$ is a hereditary shape equivalence. If $D \in \widehat{C_{0}}$, then

$$
\text { ind } \pi(D \times \widetilde{Y})=\operatorname{ind}(\pi \circ \phi(D \times \widehat{X}))
$$

and the space $\pi \circ \phi(D \times \widehat{X})$ is a closed subset of a countable dimensional space which is a cell-like image of $C \times \widehat{X}$. Thus

$$
\operatorname{ind}(\pi(D \times \tilde{Y})) \leq \eta(C \times \widehat{X}) .
$$

By our assumption $\eta(C \times \widehat{X})<\omega_{1}$.

Added in proof. It can be shown that the converse of Proposition 4.5 is also valid and that the answer to Question 4.4 is no, [14].

\section{REFERENCES}

1. F. D. Ancel, The role of countable dimensionality in the theory of cell-like relations, Trans. Amer. Math. Soc. 287 (1985), 1-40.

2. C. Bessaga and A. Pełczyński, Selected topics in infinite-dimensional topology, PWN, Warsaw, 1975.

3. K. Borsuk, Shape theory, PWN, Warsaw, 1975.

4. A. N. Dranišnikov, On a problem of P. S. Alexandrov, Mat. Sb. 135 (1988), 551-557.

5. J. Dydak and J. Segal, Shape theory: An introduction, Lectures Notes in Math., vol. 688, Springer-Verlag, Berlin, 1978.

6. R. Engelking and E. Pol, Countable dimensional spaces. A survey, Dissertationes Math. 216 (1983), 1-45.

7. W. E. Haver, Mappings between ANR's that are fine homotopy equivalence, Pacific J. Math. 58 (1975), 457-461. 
8. D. W. Henderson, A lower bound for transfinite dimension, Fund. Math. 64 (1968), 167-173.

9. J. P. Henderson and J. J. Walsh, Examples of cell-like decompositions if the infinite dimensional manifolds $\sigma$ and $\Sigma$, Topology Appl. 16 (1983), 143-154.

10. G. Kozlowski, Images of $A N R$ 's, unpublished manuscript.

11. J. van Mill, Infinite-dimensional topology, prerequisites and introduction, North-Holland, Amsterdam, 1989.

12. R. Pol, On classification of weakly infinite dimensional compacta, Fund. Math. 116 (1983), 169-188.

13. J. E. West, Open problems in infinite-dimensional topology, Open Problems in Topology (J. van Mill and G. M. Reed, eds.), North-Holland, Amsterdam, 1990, pp. 523-597.

14. J. J. Dijkstra and J. Mogilski, in preparation.

(J. J. Dijkstra, J. Mogilski) Department of Mathematics, University of Alabama, Tuscaloosa, Alabama, 35487-0350

Current address: J. Mogilski: Department of Mathematics, Bradley University, Peoria, Illinois 61625

E-mail address J.J. Dijkstra:jdijkstr@ualvm.bitnet

E-mail address: jdijkstr@ualvm.bitnet

E-mail address J. Mogilski: jmogilsk@bradley.edu

(J. van Mill) Faculteit Wiskunde en Informatica, Vrije Universiteit, Postbus 7161, 1007 MC AMSTERdaM, The NeTHERLANDS

E-mail address: vanmill@cs.vu.nl.bitnet 